\title{
ENVIRONMENTAL RESPONSIVE HYDROGELS: A NOVEL APPROACH IN DRUG DELIVERY SYSTEM
}

\author{
${ }^{1}$ Anish Kumar Gupta*, ${ }^{2}$ Abdul Wadood Siddiqui \\ ${ }^{1}$ Micro Labs ltd, Baddi, Himachal Pradesh, INDIA \\ ${ }^{2}$ Department of Pharmacy, Lloyd Institute of Management \& Technology, Greater Noida, INDIA \\ *Corresponding Author's E-Mail: anishpharma02@yahoo.co.in, Mob. +91-9312621718 \\ Received 23 Dec 2011; Revised 20 Jan 2012; Accepted 20 Jan 2012, Available online 20 Jan 2012
}

\begin{abstract}
Hydrogels are three dimensional crosslinked hydrophilic polymeric networks which has the capability to imbibe water or biological fluids. Environmentally responsive hydrogels have the ability to respond to change in their external environment. They can exhibit dramatic changes in their swelling behaviour, network structure, permeability and mechanical strength in response to change in ${ }_{\mathrm{p}} \mathrm{H}$ or ionic strength of the surrounding fluid or temperature. Other hydrogels have the ability to respond to applied electric or magnetic field or to change in concentration of glucose. Environmental responsive hydrogels for drug delivery application also requires biocompatibility. The chemical structure of the polymer may also affect the swelling ratio of hydrogels. Hydrogels containing hydrophilic groups swell to a higher degree compared to those containing hydrophobic groups. Swelling of environmentally responsive hydrogels can be affected by changes in the temperature of the swelling media, ionic strength and ${ }_{\mathrm{p}} \mathrm{H}$

Key Words: Hydrogels, Environmental responsive hydrogels, Drug delivery, Network structure.
\end{abstract}

\section{INTRODUCTION: ${ }^{1-3}$}

The goal of any drug delivery system is to provide a therapeutic amount of drug to the proper site in the body to promptly achieve and then maintain the desired drug concentration. This idealized objective points to the two aspects most important to drug delivery, namely, spatial placement and temporal delivery of a drug. Spatial placement relates to targeting of a drug to a specific organ or tissue, while the temporal delivery refers to controlling the rate of drug delivery to target tissue. An appropriately designed sustained-release drug delivery system can be a major advance towards solving these two problems.

Man has always been plagued with ailments and diseases of both the body and the mind. However dedicated research from scientists all over the world has made it possible to treat, prevent and eradicate many of these diseases that plague man. The field of pharmaceutical science has been developing steadily over the years, and has today become invaluable in helping to keep us healthy and prevent disease. An avenue of research that has progressed a great deal in the past few decades is the treatment of diseases via biomolecules such as drugs, proteins etc. Initially these could only be administered in limited manner, due to limitations of drug delivery through harmful environments in the body. Thus limited mobility reduced the effectiveness of administered drugs. Progress came with the development of biomaterial carriers which could be encapsulated, or immobilized with drugs, allowing the drug to safely reach the required site without harm. These carriers allowed for the release of drug in sites which were previously inaccessible.

These are a class of materials known as Hydrogels. These three dimensional polymer matrices are capable of imbibing large amounts of water, and biological fluids.
This property of hydrogels is the reason behind its varied applications ranging from food additives to pharmaceuticals and clinical applications. Synthetic hydrogels prepared from a varied range of monomers have found many applications especially in tissue-engineering scaffolds, as carriers for implantable devices, and drug delivery devices. Out of all these applications, at the forefront of this research are hydrogel based drug delivery devices. Synthetic hydrogels provide an effective and controlled way in which to administer protein and peptide based drugs for treatment of a number of diseases.

Stimuli sensitive hydrogels have enormous potential in various applications. Some environmental variables, such as low ${ }_{\mathrm{p}} \mathrm{H}$ and elevated temperature, are found in the body. For this reason either ${ }_{\mathrm{p}} \mathrm{H}$ sensitive or temperature sensitive hydrogels can be used for site specific controlled drug delivery. Hydrogels that are responsive to specific molecules, such as glucose or antigens, can be used as biosensors as well as drug delivery system. Environmentally sensitive hydrogels for drug delivery application also require biocompatibility.

\section{PROPERTIES OF HYDROGELS: ${ }^{4}$}

Hydrogels are water swollen polymer matrices, with a tendency to imbibe water when placed in aqueous environment. This ability to swell, under biological conditions, makes it an ideal material for use in drug delivery and immobilization of proteins, peptides, and other biological compounds. Due to their high water content, these gels resemble natural living tissue more than any other type of synthetic biomaterial. These networks, have a three dimensional structure, crosslinked together either physically (entanglements, crystallites), or chemically (tie-points, junctions). This insoluble 
crosslinked structure allows immobilization of active agents, biomolecules effectively, and allows for its release in well-defined specific manner. Thus the hydrogels biocompatibility and crosslinked structure are responsible for its varied applications.

\section{Mechanical properties:}

For non biodegradable applications, it is essential that the carrier gel matrix maintain physical and mechanical integrity. Mechanical stability of the gel is, therefore, an important consideration when designing a therapeutic system. For example, drugs and other biomolecules must be protected from the harmful environments in the body such as, extreme ${ }_{\mathrm{p}} \mathrm{H}$ environment before it is released at the required site. To this end, the carrier gel must be able to maintain its physical integrity and mechanical strength in order to prove an effective biomaterial. The strength of the material can be increased by incorporating crosslinking agents, co-monomers, and increasing degree of crosslinking. There is however an optimum degree of crosslinking, as a higher degree of crosslinking also leads to brittleness and less elasticity. Elasticity of the gel is important to give flexibility to the crosslinked chains, to facilitate movement of incorporated bioactive agent. Thus a compromise between mechanical strength and flexibility is necessary for appropriate use of these materials.

\section{Biocompatible properties:}

It is important for synthetic materials, such as hydrogels, to be biocompatible and nontoxic in order for it to be a useful biomedical polymer. Most polymers used for biomedical application must pass a cytotoxicity and in-vivo toxicity tests. Most toxicity problems associated with hydrogels arise due to unreacted monomers, oligomers and initiators that leach out during application. Thus an assessment of the potential toxicity of all materials used for fabrication of gel is an integral part of determining suitability of the gel for biological applications. To lower chances of toxic effects, the use of initiators is being eliminated, with the advent of gamma irradiation as polymerization technique. Steps are also taken to eliminate contaminants from hydrogels, by repeated washing and treatment. Also, kinetics of polymerization has been studied, so as to achieve higher conversion rates, and avoid unreacted monomers and side products.

\section{CLASSIFICATION OF HYDROGELS: ${ }^{5}$}

Hydrogels can be classified as neutral or ionic, based on the nature of side groups. In neutral hydrogels, the driving force for swelling is due to the water-polymer thermodynamic mixing contribution to the overall free energy, along with elastic polymer contribution. The swelling of ionic hydrogels is also affected by the ionic interactions between charged polymers and free ions. Ionic hydrogels containing ionic groups, such as carboxylic acid, imbibe larger amount of water, because of its increased hydrophilicity. Examples of such gels are poly (acrylic acid), and polyamines. Hydrogels are also classified as homopolymers or copolymers, based on the method of preparation. Hydrogels can be classified based on the physical structure of the network as amorphous, semicrystalline, hydrogen bonded structures, supermolecular structures and hydrocolloidal aggregates. An important class of hydrogels are the stimuli responsive gels. These gels show swelling behaviour dependent on their physical environment. These gels can swell, or deswell in response to changes in $\mathrm{pH}$, temperature, ionic strength, and electromagnetic radiation. These properties allow for usage in a number of applications, such as separation membranes, biosensors, artificial muscles, and drug delivery devices.

\section{Stimuli responsive gels: ${ }^{6}$}

Stimuli sensitive hydrogels have enormous potential in various applications. Some environmental variables, such as low $\mathrm{pH}$ and elevated temperature, are found in the body. For this reason either $\mathrm{pH}$ sensitive or temperature sensitive hydrogels can be used for site specific controlled drug delivery. Hydrogels that are responsive to specific molecules, such as glucose or antigens, can be used as biosensors as well as drug delivery system (Figure 1).

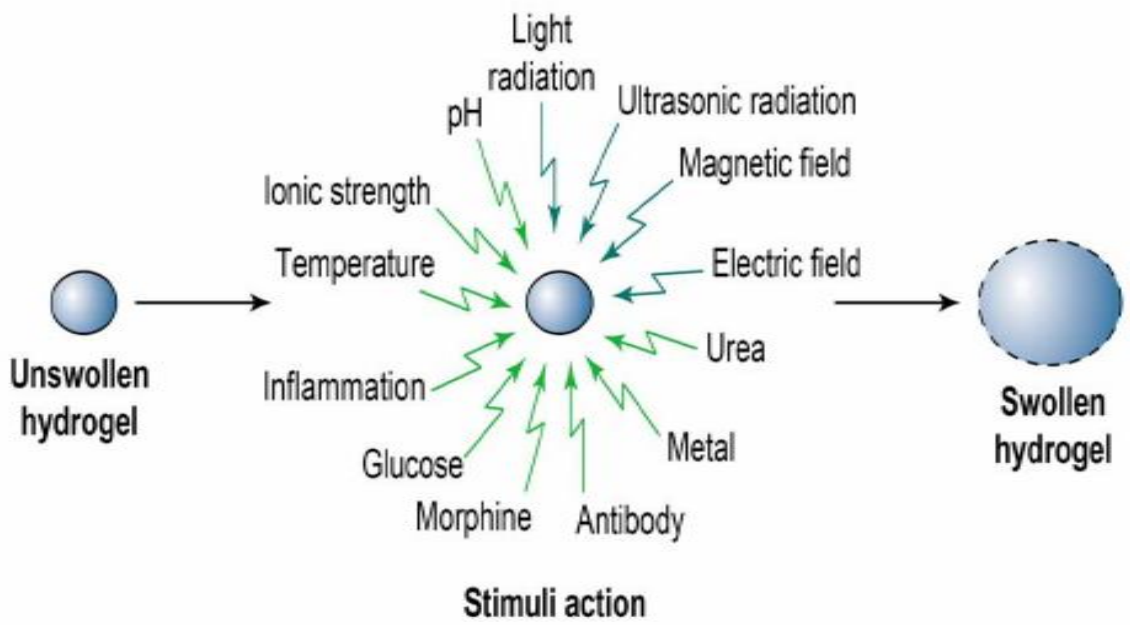

Figure 1: Stimuli responsive swelling of hydrogels 


\section{Temperature sensitive hydrogels:}

\subsection{Polymer structure:}

Many polymers exhibit a temperature responsive phase transition property. The common characteristic of temperature sensitive polymers is the presence of hydrophobic groups, such as methyl, ethyl and propyl groups. Of the many temperature sensitive polymers, poly (N-iso-propylacrylamide) (PNIPAAm) is probably the most extensively used. Poly (N, N-diethylacrylamide) (PDEAAm) is also widely used because of its lower critical solution temperature (LCST) in the range of 25$32^{\circ} \mathrm{C}$, close to the body temperature.

\subsection{Properties of temperature sensitive hydrogels:}

Most polymers increase their water solubility as the temperature increases. Polymers with LCST, however, decrease their water solubility as the temperature increases above the LCST. This type of swelling behaviour is known as inverse (or negative) temperature dependence. The inverse temperature dependent hydrogels are made of polymer chains that either posses moderately hydrophobic groups (if too hydrophobic, polymer chains would not dissolve in water at all) or contain mixture of hydrophilic and hydrophobic segments.

At lower temperatures, hydrogen bonding between hydrophilic segments of the polymer chain and water molecules are dominates, leading to enhanced dissolution in water. As the temperature increases, however, hydrophobic interactions among hydrophobic segments become strengthened, while hydrogen bonding becomes weaker. The net result is shrinking of the hydrogels due to inter-polymer chain association through hydrophobic interactions. The LCST can be changed by adjusting the ratio of hydrophilic and hydrophobic segment of the polymer. If the polymer chains in hydrogels are not covalently cross linked, temperature sensitive hydrogels may undergo sol-gel phase transitions, instead of swellingshrinking transitions. Polymers that show this type of behaviour are block copolymers of PEO and PPO. Temperature sensitive hydrogels can also be made using temperature-sensitive crosslinking agents.

\section{$2{ }_{\mathrm{p}} \mathrm{H}$ sensitive hydrogels:}

\subsection{Polymer structures:}

All the ${ }_{\mathrm{p}} \mathrm{H}$-sensitive polymers contain pendant acidic (e.g. carboxylic and sulphonic acid) or basic (e.g. ammonium) groups that either accept or release protons in response to change in environmental ${ }_{\mathrm{p}} \mathrm{H}$. The polymers with a large number of ionizable groups are known as polyelectrolytes. Poly acrylic acid (PAA) becomes ionized at high ${ }_{\mathrm{p}} \mathrm{H}$, while poly (N, N'-diethylaminoethyl methacrylate) (PDEAEM) becomes ionized at low ${ }_{\mathrm{p}} \mathrm{H}$. Cationic polyelectrolyte, such as PDEAEM, dissolve more or swell more if crosslinked at low ${ }_{\mathrm{p}} \mathrm{H}$ due to ionization. On the other hand, polyanions, such as PAA, dissolve more at high ${ }_{\mathrm{p}} \mathrm{H}$.

\subsection{Properties of $\mathrm{p} H$-sensitive hydrogels:}

Hydrogels made of crosslinked polyelectrolyte's display big differences in swelling properties depending on the ${ }_{\mathrm{p}} \mathrm{H}$ of the environment. The pendant acidic or basic groups on polyelectrolyte undergo ionization just like acidic or basic groups of monoacids or monobases. The presence of ionizable groups on polymer chains result in swelling of the hydrogels much beyond that can be achievable by nonelectrolyte polymer hydrogels. Swelling of polyelectrolyte hydrogels is mainly due to the electrostatic repulsion among charges present on the polymer chain.

Different co-monomers provide different hydrophobicity to the polymer chain, leading to different ${ }_{\mathrm{p}} \mathrm{H}$-sensitive behaviour. Hydrogels made of poly (methacrylic acid) (PMA) grafted with poly (ethylene glycol) (PEG) have unique ${ }_{\mathrm{p}} \mathrm{H}$-sensitive properties. At low ${ }_{\mathrm{p}} \mathrm{H}$, the acidic protons of the carboxyl groups of PMA interact with the ether oxygen of PEG through hydrogen bonding, and such complexation results in shrinkage of hydrogels. As the carboxyl groups of PMA become ionized at high ${ }_{p} \mathrm{H}$, the resulting decomplexation leads to swelling of hydrogels (Figure 2)

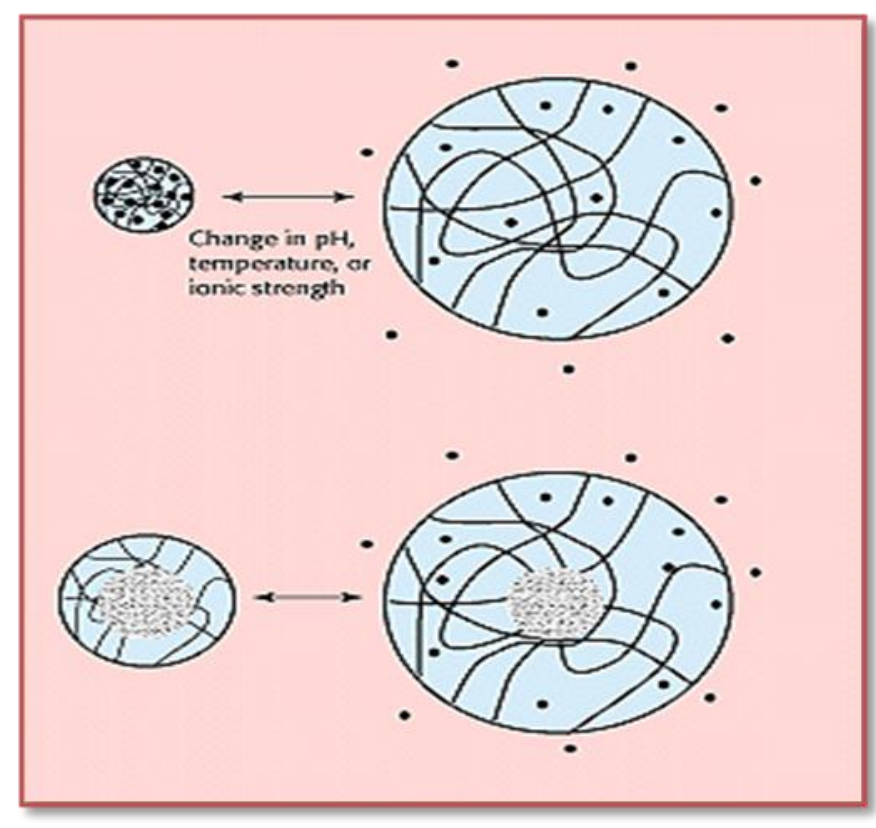

Figure 2: Environmental sensitive swelling-controlled release system

\section{Glucose sensitive hydrogels:}

One of the most challenging problems in controlled drug delivery area is the development of self-regulated (modulated) insulin delivery system. Delivery of insulin is different from delivery of other drugs, since insulin has to be delivered in an exact amount at the exact time of need. Thus, self-regulated insulin delivery systems require the glucose sensing ability and an automatic shut-off mechanism. Many hydrogel systems have been developed for modulating insulin delivery, and all of them have a glucose sensor built into the system.

Glucose oxidase is probably the most widely used enzyme in glucose sensing. It oxidizes glucose to gluconic acid, resulting in $\mathrm{a}_{\mathrm{p}} \mathrm{H}$ change of the environment. This makes it possible to use different types of ${ }_{\mathrm{p}} \mathrm{H}$-sensitive hydrogels for modulated insulin delivery. 


\section{Electric signal-sensitive hydrogels:}

Electric current can also be used as an environmental signal to induce responses of hydrogels. Hydrogels sensitive to electric current are usually made of polyelectrolytes, as are ${ }_{\mathrm{p}} \mathrm{H}$ sensitive hydrogels. Sometimes the hydrogels show swelling on one side and deswelling on other side, resulting in bending of the hydrogels. Partially hydrolyzed polyacrylamide hydrogels which are in contact with both the anode and cathode electrodes undergo volume collapse by an infinitesimal change in electric potential across the gel.

\section{Light-sensitive hydrogels:}

Light sensitive hydrogels have potential application in developing optical switches, display units, and ophthalmic drug delivery devices. Since the light stimulus can be impose instantly and delivered in specific amounts with high accuracy, light sensitive hydrogels may posses special advantage over others. For example, sensitivity of temperature controlled hydrogels rate limited by thermal diffusion, while $\mathrm{pH}$ sensitive hydrogels can be limited by hydrogen ion diffusion. Light sensitive hydrogels can be separated in to UV-sensitive and visible light sensitive hydrogels.

The UV-sensitive hydrogels were synthesized by introducing a leuco derivative molecule, bis (4-dimethylamino) phenylmethyl leucocynide, in to the polymer network. Visible light sensitive hydrogels were prepared by introducing a light sensitive chromophore (e.g. tri sodium salt of copper chlorophyllin) to poly ( $\mathrm{N}$-isopropylacrylamide).

\section{Pressure sensitive hydrogels:}

The concept that hydrogels may undergo pressure induced volume phase transition came from thermodynamic calculation based on uncharged hydrogel theory. According to the theory, hydrogels which are collapsed at low pressure would expand at higher pressure. Experiments with poly ( $\mathrm{N}$-iso-propylacrylamide) hydrogels confirmed this prediction. The degree of swelling of poly ( $\mathrm{N}$-iso-propylacrylamide) hydrogels increased under hydrostatic pressure when the temperature is close to its LCST.

\section{Specific ion sensitive hydrogels:}

Little or no effect of salt concentration on swelling behaviour is expected for neutral hydrogels. A nonionic poly (N-iso-propylacrylamide) hydrogel, however, showed a sharp volume phase transition at a critical concentration of sodium chloride in aqueous solution. Below the LCST, the water content of the hydrogel is a strong function of the sodium chloride concentration.

The gel collapse sharply at a critical sodium chloride concentration, this concentration is also found to be temperature dependent. Increasing temperatures leads to a corresponding decrease in the critical concentration of sodium chloride. The LCST of the hydrogel appeared to be lowered by increasing the chloride concentration. This unique phase transition behaviour could be applicable to making chloride ion sensitive biosensors.

\section{Specific antigen-responsive hydrogels:}

For some biomedical applications, it is highly desirable and useful to develop a material or devices, which can response to specific proteins. Sol-gel phase reversible hydrogels were prepared based on antigen-antibody interactions. The concept is same as that used in glucose sensitive phase reversible hydrogels. A semiinterpenetrating network hydrogels was prepared by grafting an antigen and a corresponding antibody to different polymer networks. The gel is formed by crosslinking interactions that occur upon antigen-antibody binding.

\section{Thrombin-induced infection responsive hydrogels:}

For release of antibiotics at the site and time of infection, PVA hydrogels loaded with grafted gentamycin were made. Gentamycin was chemically attached to the polymer backbone through peptide linkers that can enzymatically degraded by thrombin. This type of approach can be applied to occlusive wound dressing and infection prone catheters, drainage bags and prostheses.

\section{MONOMERS USED FOR FABRICATION OF HYDROGELS: ${ }^{7}$}

The monomers used for fabrication of these biocompatible hydrogels have expanded from a handful of choices, to several novel materials with tailor-made properties suited to particular applications. The first synthesis of hydrogel was done by Wichterle and Lin using PHEMA (poly (hydroxyethyl methacrylate)) as the monomer. Depending upon the application, hydrogel monomers are chosen according to their properties, ease of delivery or encapsulations, as well as cost and availability. One of the most traditional monomers used for drug delivery of proteins is biodegradable PLGA (polymers of lactic and glycolic acid).

Table 1: Monomers used for biomaterial synthesis. ${ }^{8}$

\begin{tabular}{|l|l|}
\hline $\begin{array}{c}\text { Monomer } \\
\text { Abbreviation }\end{array}$ & \multicolumn{1}{|c|}{ Monomer } \\
\hline HEMA & Hydroxyethyl methacrylate \\
\hline HEEMA & Hydroxyethoxyethyl methacrylate \\
\hline HDEEMA & Hydroxydiethoxyethyl methacrylate \\
\hline MEMA & Methoxyethyl methacrylate \\
\hline MEEMA & Methoxyethoxyethyl methacrylate \\
\hline MDEEMA & Methoxydiethoxyethyl methacrylate \\
\hline EGDMA & Ethylene glycol dimethacrylate \\
\hline NVP & N-vinyl-2-pyrrolidone \\
\hline NIPAAm & N-isopropyl AAm \\
\hline Vac & Vinyl acetate \\
\hline AAc & Acrylic acid \\
\hline MAA & Methacrylic acid \\
\hline HPMA & N-(2-hydroxypropyl) methacrylamide \\
\hline EG & Ethylene glycol \\
\hline PEGA & PEG acrylate \\
\hline PEGMA & PEG methacrylate \\
\hline PEGDA & PEG diacrylate \\
\hline PEGDMA & PEG dimethacrylate \\
\hline
\end{tabular}

However these hydrophobic materials have a tendency to denature protein as well as cause inflammation due to 
degradation. These problems were overcome when researchers turned towards hydrophilic monomers. Monomers such as acrylic acid, polyethylene glycol, and methacrylic acid are all materials used in therapeutic applications. PNIPAAm poly (Nisopropylacrylamide), PVA (polyvinyl alcohol), is all synthesized by new preparation techniques, for distinct applications. Table provides a list of popular monomers used for biomaterial synthesis (Table 1).

\section{APPLICATIONS OF HYDROGELS: ${ }^{9}$}

Water- swollen crosslinked hydrogels have varied applications in fields such as food additives, pharmaceutical as well as biomedicine. The pioneering work on crosslinked HEMA hydrogels was done by Wichterle and Lim in 1954. From their research, and discovery of the hydrophilic and biocompatible properties of hydrogels, there emerged a new class of hydrogel technologies based on biomaterial application. Lim and Sun in 1980 demonstrated the successful use of calcium alginate microcapsules for cell encapsulation. Later natural polymers such as collagen, and shark cartilage were incorporated into hydrogels as wound dressings. Natural and synthetic polymers are used for encapsulation of cells, as well as encapsulation of islets in a semi permeable membrane. Hydrogels have been used to prevent adhesions and prevent thrombosis after surgery, and as cell adhesion resistant surfaces. Microfabricated hydrogel arrays are also used for biosensing.

Hydrogels now play an important role in tissue engineering scaffolds, biosensor and BioMEMS devices and drug carriers. Among these applications, hydrogelbased drug delivery devices have become a major area of study, and several commercially available products are already in the market. Proteins, peptides, DNA based drugs can all be delivered via hydrogel carrier devices.

\section{Hydrogels in Drug Delivery: ${ }^{10}$}

Localized drug delivery can be achieved by introducing the drug directly at the target site. The major class of biomaterials considered as implantable drug delivery systems are hydrogels. These hydrophilic networks are capable of absorbing great amounts of water while maintaining structural integrity. Their structural similarity to the extracellular matrix makes it biocompatible. These synthetic polymers have generated wide interests and are now at the forefront of drug delivery research.

In order to incorporate a preformed gel into the body, an opening must be created, with at least the same dimension as that of the gel. This leads to potential risk and discomfort to the patient. Thus focus has shifted to developing injectable materials with ability to form three dimensional matrices under physiological matrices. This in-situ formation can be achieved through specific chemical crosslinking reactions. Gel structuring is triggered by environmental stimuli $(\mathrm{pH}$, temperature, solvent exchange etc). Synthetic hydrogels, with their ability to imbibe water, flexibility, and biocompatibility, are ideal carriers for the development for novel pharmaceutical formulations and for the delivery of drugs, proteins, and as targeting agents for drug delivery. The network structure and the nature of components play a key role in the diffusional behaviour, molecular mesh size changes, and stability of the incorporated bioactive agent. The use of hydrogels allows not only delivery of drugs, but also controlled release, in the manner required by the pharmaceutical scientists. For example, drugs can be delivered only when needed, may be directed to specific site, and can be delivered at specific rates required by the body.

\section{Properties useful in drug delivery: ${ }^{11}$}

Hydrogels possess several properties that make them an ideal material for drug delivery. First, hydrogels can be tailored to respond to a number of stimuli. This enables sustained drug delivery corresponding to external stimuli such as $\mathrm{pH}$ or temperature. These $\mathrm{pH}$ sensitive gels are useful in oral drug delivery as they can protect proteins in the digestive track. $\mathrm{pH}$ responsiveness is also useful for lysosomal escape during gene delivery. Second, Hydrogels can also be synthesized to exhibit bioadhesiveness to facilitate drug targeting, especially through mucus membranes, for non-invasive drug administration. Finally, Hydrogels also have a "stealth" characteristic in-vivo circulation time of delivery device by evading the host immune response and decreasing phagocytic activity.

\section{Applications of hydrogels in drug delivery: ${ }^{12}$}

Advances in recombinant protein technology have identified several protein and peptide therapeutics for disease treatment. However, the problem which plagued researchers was how to effectively deliver these biomolecules. Due to their large molecular weight, and three dimensional structure, the most commonly used route for drug administration is by intravenous or subcutaneous injection. Unfortunately proteins and peptides are prone to proteolytic degradation, thus they experience short plasma circulation times and rapid renal clearance, leading to multiple daily injections or increased dosage in order to maintain the required drug therapeutic levels . Multiple injections are difficult for the patient, while high doses might be toxic, and induce serious immune response. Hydrophobic polymeric controlled release formulations, such as PLGA, offer a sustained release mechanism in which drug release rates can manipulated by changing polymer molecular weight and composition. These polymers however induce adverse effects to the encapsulated proteins or peptides during network preparation and delivery, as well as trigger the immune response. Hydrophilic hydrogels, on the other hand, provide relatively mild network fabrication technique and drug encapsulation conditions, making them the ideal material for use in drug delivery. Thus hydrogels are primarily used for encapsulation of bioactive materials and their subsequent controlled release.

If designed properly, hydrogels can be used in a variety of applications such as sustained, targeted, or stealth biomolecules delivery. Hydrogel based delivery devices can be used for oral, ocular, epidermal and subcutaneous application. The Figure 3 below indicates various sites that are available for the application of hydrogels for drug delivery. These applications are discussed in detail below. 
Table 2: Biomedical applications of synthetic hydrogels

\begin{tabular}{|l|l|l|}
\hline Coatings & Homogeneous Materials & Devices \\
\hline Sutures & Electrophoresis gels & Enzyme therapeutic \\
Catheters & Contact lenses & systems \\
Blood detoxicants & Artificial corneas & Artificial organs \\
Sensors & Oestrous -Induces & Sustained drug delivery systems \\
Vascular grafts & Soft tissue substitutes & \\
Electrophoresis cells & Burn dressings & \\
Cell structure substrates & Dentures & \\
& Ear drum plugs & \\
& Haemodialysis & \\
& membranes & \\
& Vitreous-humour & \\
& replacements & \\
& & \\
\end{tabular}

\subsection{Drug delivery in the GI tract:}

The ease of administration of drugs, and the large surface area for absorption makes the GI tract most popular route for drug delivery. It is however, also a very complex route, so that versatile approaches are needed to deliver drugs for effective therapy. Hydrogel-based devices can be designed to deliver drugs locally to specific sites in the GI tract.

For example, Patel and Amiji, ${ }^{13}$ proposed stomachspecific antibiotic drug delivery systems for the treatment of Helicobacter pylori infection in peptic ulcer disease. They developed cationic hydrogels with ${ }_{\mathrm{p}} \mathrm{H}$ sensitive swelling and drug release properties for antibiotic delivery in the acidic environment of the stomach. There are still many drawbacks for peroral delivery of peptides and proteins to GI tract, like protein inactivation by digestive enzymes in the GI tract and poor epithelial permeability of the drugs. However certain hydrogels may overcome some of these problems by appropriate molecular design or formulation. For example Akiyama ${ }^{3}$ reported novel peroral dosage forms of hydrogel formulations with protease inhibitory activities.

Recently oral insulin delivery using ${ }_{\mathrm{p}} \mathrm{H}$ responsive complexation hydrogels was reported. ${ }^{14}$ The hydrogels used were crosslinked copolymers of PMMA with graft chains of polyethylene glycol. These hydrogels protect the insulin in the harsh, acidic environment of the stomach before releasing the drug in the small intestine. The colonic region has also been considered as a possible absorption site for orally administered proteins and peptides, mostly due to a lower proteolytic activity in comparison to that in the small intestine. Several hydrogels are currently being investigated as potential devices for colon-specific drug delivery. These include chemically or physically crosslinked polysaccharides such as dextran, guar gum and insulin. They are designed to be highly swollen or degraded in the presence of colonic enzymes or micro flora, providing colon-specificity in drug delivery.

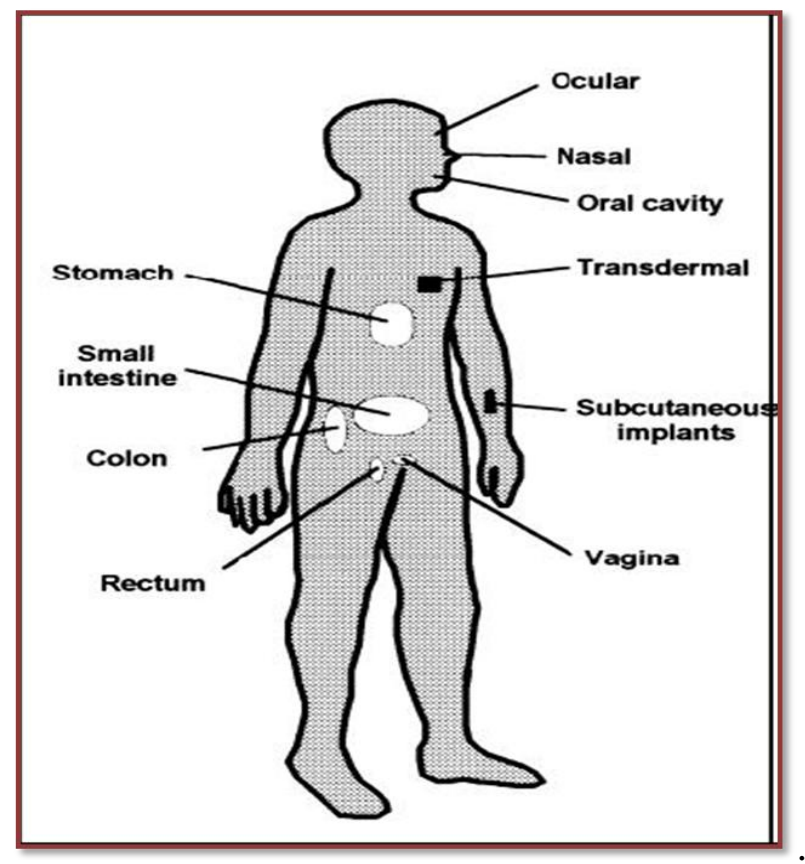

Figure 3: Tissue locations applicable for hydrogel based

drug delivery systems

\subsection{Rectal delivery:}

This route has been used to deliver many types of drugs for treatment of diseases associated with the rectum, such as haemorrhoids. This route is an ideal way to administer drugs suffering heavy first-pass metabolism. There are however, some drawbacks associated with rectal delivery. For example, due to discomfort arising from given dosage forms, there is substantial variability in patient's acceptance of treatment. Also, if drugs diffusing out of the suppositories are delivered in an uncontrolled manner, they are unable to be retained at a specific position in the rectum, and tend to migrate upwards to the colon. This leads to variation of availability of drugs, especially those that undergo extensive first-pass elimination. Hydrogels offer a way in which to overcome these limitations, provided that the hydrogels show bioadhesive properties. It 
was reported that increased bioavailability of propranolol subject to extensive first-pass metabolism was observed by adding certain mucoadhesive polymeric compounds to poloxamer based thermally gelling suppositories. ${ }^{19}$ The polymeric compounds tested were polycarbophil and sodium alginate.

\subsection{Ocular delivery:}

Drug delivery to the eye is difficult due to its protective mechanisms, such as effective tear drainage, blinking, and low permeability of the cornea. Thus, eye drops containing drug solution tends to be eliminated rapidly from the eye and the drugs show limited absorption, leading to poor ophthalmic bioavailability. Due to the short retention time, a frequent dosing regimen is necessary for required therapeutic efficacy. These challenges have motivated researchers to develop drug delivery systems that provide prolonged residence time. The earlier dosage forms, such as suspension and ointments could be retained in the eye, but sometimes gave patients an unpleasant feeling because of the nature of solids and semisolids. Hydrogels, because of their elastic properties can represent an ocular drainageresistant device. In-situ forming hydrogels are attractive as an ocular drug delivery system because of their facility in dosing as a liquid, and long term retention property as a gel after dosing. Cohen et al. ${ }^{7}$ developed an in-situ gelling system of alginate with high gluconic acid contents for the ophthalmic delivery of pilocarpine. This system extended the duration of the pilocarpine to $10 \mathrm{hr}$, compared to $3 \mathrm{hr}$ when pilocarpine nitrate was dosed as a solution.

\subsection{Transdermal delivery:}

Drug delivery to the skin has been generally used to treat skin diseases or for disinfection of the skin. In recent years, however a transdermal route for the delivery of drugs has been investigated. Swollen hydrogels can be delivered for long duration and can be easily removed. These hydrogels can also bypass hepatic first-class metabolism, and are more comfortable for the patient. Hydrogel based delivery devices have been proposed by Sun et $a l^{15}$ such as composite membranes of crosslinked PHEMA with a woven polyester support. Also hydrogels have been reported which have been obtained by the copolymerization of bovine serum albumin (BSA) and PEG. ${ }^{16}$ These hydrogels can be used as controlled release devices in the field of wound dressing.

Current research in this field is now focused on electrically-assisted delivery using iontophoresis and electroporation. Hydrogel-based formulations are being looked at for transdermal iontophoresis to obtain enhanced permeation of products in question such as, hormones and nicotine. ${ }^{13}$

\subsection{Subcutaneous delivery:}

Among the varied possible pharmaceutical applications of hydrogels, the most substantial application is probably in implantable therapeutics. Implantable devices that are subcutaneously inserted tend to illicit immune response of the body, leading to inflammation, carcinogenicity and immunogenicity. Thus biocompatibility becomes a major issue, and all implantable materials must be compatible with the body. Hydrogels are an ideal candidate for implantable materials. They have high water content, environment similar to biological tissue, making them relatively biocompatible. They also have other properties which make them a viable choice: ${ }^{17}$

$>$ Minimal mechanical irritation upon in-vivo implantation due to their soft, elastic properties.

$>$ Prevention of protein absorption and cell adhesion arising from the low interfacial tension between water and hydrogels.

$>$ Broad acceptability for individual drugs with different hydrophilicities and molecular sizes.

$>$ Manipulate crosslinking density and swelling for release of incorporated drug in specific manner. Thus hydrogels are an ideal material to be used for delivery of proteins and peptides.

Hydrogel formulations for subcutaneous delivery of anticancer drugs have been proposed. For example, crosslinked PHEMA was applied to cyratabine (Ara-C). Current studies on implantable hydrogels are leading towards the development of biodegradable systems, which don't require surgical removal once the drug has been administered.

Biodegradable PEG hydrogels are now at the forefront of this research and several novel systems have been developed. One type is synthesized via a polycondensation reaction between functional PEG acids and branched PEG polyols. Another type is PEG based hydrogels having functional groups in which the protein drugs can be covalently attached to the gel network via ester linkages. In this case, the release of the immobilized proteins would be controlled by the hydrolysis of ester linkage between the gel and protein, followed by diffusion of protein, and degradation of gel.

\section{HYDROGELS AS BIOMATERIALS: ${ }^{18}$}

The field of biomedical research has advanced rapidly in the past several years, mainly as a result of attempts to replace body tissues with natural or synthetic biomaterials. Success in the application of biomaterials is strictly confined to their biocompatibility. The term biocompatibility is referred to as the appropriate biological performance, both local and systematic of a given biomaterial in a specific application. An appropriate response of the biomaterial for its particular application would be referred to as an inert or positive interaction with the host. One promising class of biomaterials in this field of research is that of polymeric hydrogels (Table 2). 


\section{REFERENCES}

1. Hoffman AS. Hydrogels for biomedical applications. Ad. Drug Del. Rev.2002, 54(1) : 3-12.

2. Peppas NA et al. Hydrogels in pharmaceutical formulations. Eur. J. Pharm. Bio. 2000, 50(1) : 27-46.

3. Peppas NA. Hydrogels in Medicine and Pharmacy. Vol. I. Fundamentals. CRC Press. Boca Raton, FL.1986, 180.

4. Lin CC and Metters AT. Hydrogels in controlled release formulations: Network design and mathematical modeling. Adv. Drug Del. Rev.2006, 58(12-13) : 1379-1408.

5. Dolbow J, Fried E, and Ji H. A numerical strategy for investigating the kinetic response of stimulus-responsive hydrogels. Computer Methods in Applied Mechanics and Engineering.1997, 194 (42-44) : 4447-4480.

6. Yong Q and Park K. Environmental-sensitive hydrogels for drug delivery, Ad. Drug Del. Rev. 2001, 53 : 321-339.

7. Wichterle $\mathrm{O}$ and Lim D. Hydrophilic gels for biological use. Nature. 1960, 185(4706) : 117-118.

8. Peppas NA and Sahlin JJ. Hydrogels as mucoadhesive and bioadhesive materials: a review. Biomaterials. 1996,

9. 17(16) : 1553-1561.

10. Chen $X$ et al. Enzymatic and chemoenzymatic approaches to synthesis of sugar-based polymer and hydrogels. Carbohydrate Polymers, 1995. 28 (1) : 15-21.

11. Nagai $T$ and Machida $Y$. Buccal delivery systems using hydrogels. Ad. Drug. Del. Rev. 1993, 11(1-2) : 179-191.

12. Achar L and Peppas NA. Preparation, characterization and mucoadhesive interactions of poly (methacrylic acid) copolymers with rat mucosa. J. Control. Release. 1994, 31 (3) : 271-276.

13. Peppas NA et al. Hydrogels in pharmaceutical formulations. Eur. J. Pharm. Bio. 2000, 50(1) : 27-46.

14. Fang JY et al. Transdermal iontophoresis of sodium nonivamide acetate: Combined effect of physical enhancement methods. Int. J. Pharm. 2002, 235 (1-2) : 95105.

15. Peppas NA. Hydrogels in Medicine and Pharmacy. Vol. I. Fundamentals. CRC Press. Boca Raton, FL. 1986, 180.

16. Sun YM et al. Composite poly (2-hydroxyethyl methacrylate) membranes as ratecontrolling barriers for transdermal applications. Biomaterials. 1997, 18(7) : 527-533.
17. Gayet JC and Fortier G. High water content BSA-PEG hydrogel for controlled release device: Evaluation of the drug release properties. J. Control. Release. 1996, 38 (2-3) : 177184.

18. Park H and Park K. Biocompatibility issues of implantable drug delivery systems. 1996, Pharm. Res. 13 (12) : 17701776.

19. Rosaik JM. Radiation formation of hydrogels for drug delivery. J. Control. Release. 1994, 31: 9-19.

20. Achar L and Peppas NA. Preparation, characterization and mucoadhesive interactions of poly (methacrylic acid) copolymers with rat mucosa. J. Control. Release. 1994, 31 (3) : 271-276.

21. Chen X et al. Enzymatic and chemoenzymatic approaches to synthesis of sugar-based polymer and hydrogels. Carbohydrate Polymers. 1995, 28 (1) : 15-21.

22. Cohen $\mathrm{S}$ et al. A novel in-situ gel forming ophthalmic drug delivery system from alginates undergoing gelation in the eye. J. Control. Release. 1997, 44 (2-3) : 201-208.

23. Peppas NA and Sahlin JJ. Hydrogels as mucoadhesive and bioadhesive materials: a review. Biomaterials. 1996, 17(16) : 1553-1561.

24. Chen X et al. Enzymatic and chemoenzymatic approaches to synthesis of sugar-based polymer and hydrogels. Carbohydrate Polymers. 1995, 28 (1) : 15-21.

25. Nagai $\mathrm{T}$ and Machida Y. Buccal delivery systems using hydrogels. Ad. Drug. Del. Rev. 1993, 11(1-2) : 179-191.

26. Achar L and Peppas NA. Preparation, characterization and mucoadhesive interactions of poly (methacrylic acid) copolymers with rat mucosa. J. Control. Release. 1994, 31 (3) : 271-276.

27. Peppas NA et al. Hydrogels in pharmaceutical formulations. Eur. J. Pharm. Bio. 2000, 50(1) : 27-46.

28. Fang JY et al. Transdermal iontophoresis of sodium nonivamide acetate: Combined effect of physical enhancement methods. Int. J. Pharm. 2002, 235 (1-2) : 95105. 